\title{
Desarrollo de competencias transversales para promover la excelencia del alumnado
}

A. Martinez García, M. Cabedo Fabrés, S. Calvet Sanz, A. Jiménez Belenguer, I. Guillén Guillamón, S. Ibánez Asensio, H. Moreno Ramón,C. Cárcel García, M. Ferrando Bataller

Universitat Politécnica de Valencia, Valencia, Spain (alfonsomg@dcom.upv.es)

\begin{abstract}
This paper describes the work performed in the last year within the Teaching Innovation Project "Development of generic skills to promote student excellence" by the team of Innovation and Educational Quality (EICE) GRIPAU at the Universitat Politècnica de València (UPV).

GRIPAU is a multidisciplinary team of teachers focused on promoting innovation and improving the quality of learning in Higher Education.

In this paper GRIPAU has implemented a teaching methodology that facilitates the acquisition of different transverse skills by students, allowing progress towards excellence, understood from the point of view of their integral development. Pilot experiences have been conducted to develop transverse skills in subjects of different degrees, establishing a common pattern of performance. A study of the starting level of students has been carried out for each skill. Then, the student has been given proper guidelines to develop the skill. Finally, students are informed about the degree of accomplishment and the level of excellence achieved after completing the subject. The result of these experiences constitutes a set of best practices for other teachers for working transverse skills in a structured and consistent way.
\end{abstract}

Keywords: Higher education, transverse skills, excellence, motivation, emotional inteligence, self-reflection. 
Desarrollo de competencias transversales para promover la excelencia del alumnado

\begin{abstract}
Resumen
Esta comunicación describe el trabajo realizado en el último año dentro del Proyecto de Innovación Docente "Desarrollo de competencias transversales para promover la excelencia del alumnado" por el Equipo de Innovación y Calidad Educativa (EICE) GRIPAU en la Universitat Politècnica de València (UPV).
\end{abstract}

GRIPAU es un equipo multidisciplinar de profesores, centrado en promover la innovación y mejorar la calidad del aprendizaje en Educación Superior.

En este trabajo GRIPAU ha puesto en práctica una metodología docente que facilita la adquisición de diferentes competencias transversales por parte del alumno, permitiéndole avanzar hacia la excelencia, entendida ésta desde el punto de vista de su desarrollo integral. Se han realizado experiencias piloto de desarrollo de competencias transversales en asignaturas de diferentes titulaciones, estableciendo una pauta común de actuación. Para cada competencia se ha realizado una valoración inicial del nivel de partida del alumnado, se ha desarrollado la competencia dando al estudiante las directrices adecuadas, y se ha evaluado el grado de consecución y el nivel de excelencia alcanzado por el alumno en dicha competencia tras cursar la asignatura. El resultado de estas experiencias constituye un conjunto de buenas prácticas para otros docentes de cómo trabajar competencias transversales de forma estructurada y coherente.

Palabras clave: Educación superior, competencias transversales, excelencia, motivación, inteligencia emocional y autoreflexión.

\title{
1. Introducción
}

El Proceso de Bolonia ha propiciado la realización de diversas reformas en Educación Superior, orientadas a adaptar la universidad a la nueva realidad social, denominada Sociedad del Conocimiento. Estas reformas se han orientado en múltiples direcciones, pero sobre todo en las metodologías docentes, en la estructura de las enseñanzas, y en la calidad de los procesos de aprendizaje. Siguiendo las directrices del proceso de Bolonia, la Universitat Politècnica de València (UPV) ha puesto en marcha múltiples iniciativas que han favorecido la modernización de sus títulos, y la introducción de las nuevas tecnologías en los procesos de aprendizaje. Según la edición del TIMES HIGHER EDUCATION WORLD UNIVERSITY RANKINGS (2014) la UPV se encuentra entre las 400 mejores universidades del mundo, siendo la única universidad tecnológica española que ha logrado posicionarse en este prestigioso ranking. 
A. Martinez García, M. Cabedo Fabrés, S. Calvet Sanz, A. Jiménez Belenguer, I. Guillén Guillamón, S. Ibánez Asensio, H. Moreno Ramón,C. Cárcel García, M. Ferrando Bataller

No obstante, aunque la calidad de la formación técnica de los estudiantes de la UPV está sobradamente reconocida, en lo que respecta a la orientación al empleo aún tenemos mucho que mejorar y aquí es dónde entran en juego las competencias transversales. (FERNÁNDEZ, 2010).

En la actualidad es cada vez más evidente que existen desajustes entre la formación universitaria y las demandas de los empleadores en cuanto a estas competencias (MARTÍN DEL PESO, 2013). Por todo ello, el desarrollo de las competencias transversales en estudios universitarios ha recibido una creciente atención en los últimos años, en los que numerosas instituciones han establecido iniciativas tratando de incorporar estas competencias como elemento de valor de sus titulaciones (FALLOWS, 2000; ALSINA, 2011).

El último estudio sobre Empleabilidad de Titulados realizado por el Observatorio de Empleo UPV (AYATS, 2010), refleja que las valoraciones de las competencias transversales que poseen los titulados universitarios cuando se incorporan a la empresa son siempre inferiores a las valoraciones de las competencias demandadas para desempeñar su puesto de trabajo. El estudio destaca que existe un déficit de competencias transversales de los titulados universitarios desde el punto de vista del empleador. Resulta destacable que más de la mitad de las competencias transversales estudiadas no alcanzan la nota media de 5. En base a estos resultados, la Universitat Politècnica de València (UPV) está embarcada actualmente en un ambicioso proyecto institucional de desarrollo, evaluación y calificación de 13 competencias transversales. Este proyecto se enmarca dentro del Plan Estratégico UPV 2015-2020 (UPV, 2014). El objetivo es mejorar la capacitación de los estudiantes para su posterior inserción laboral. A nivel práctico, se han establecido en este proyecto institucional las siguientes competencias transversales:

C1. Comprensión e integración.

C2. Aplicación y pensamiento práctico.

C3. Análisis y resolución de problemas.

C4. Innovación, creatividad y emprendimiento.

C5. Diseño y proyecto.

C6. Trabajo en equipo y liderazgo.

C7. Responsabilidad ética, medioambiental y profesional.

C8. Comunicación efectiva.

C9. Pensamiento crítico.

C10. Conocimiento de problemas contemporáneos.

C11. Aprendizaje permanente.

C12. Planificación y gestión del tiempo.

C13. Instrumental específica.

De todas estas competencias, según el estudio sobre Empleabilidad de Titulados de 2010, las más demandadas por las empresas son la capacidad para trabajar en equipo (C6), la capacidad

(c) EY-NC-ND 2015, Universitat Politècnica de València

Congreso In-Red (2015) 
para adquirir conocimientos (C11), la capacidad para utilizar herramientas informáticas (C13), y la capacidad para usar el tiempo de forma efectiva (C12). Por tanto, si desde la UPV queremos formar profesionales orientados al empleo y capacitados para cubrir las demandas del mundo laboral, es necesario esforzarse por mejorar el desarrollo de competencias transversales entre nuestro alumnado. Aunque, siendo realistas, lo cierto es que existe cierta reticencia por parte del profesorado a trabajar competencias transversales desde el aula, seguramente debido al gran desconocimiento por su parte respecto a cómo desarrollar y evaluar estas competencias.

En este contexto, el Equipo de Innovación y Calidad Educativa (EICE) de la UPV GRIPAU ha llevado a cabo el proyecto de innovación docente "Desarrollo de competencias transversales para promover la excelencia del alumnado”. GRIPAU (Grupo Interdisciplinario Para el Aprendizaje Universitario), que está compuesto por nueve profesores que llevan a cabo su docencia en diversas disciplinas como agronomía, tecnología de los alimentos, arquitectura, forestales-ambientales y telecomunicaciones. En este proyecto GRIPAU pretende llevar a cabo una serie de experiencias piloto de desarrollo y evaluación de competencias transversales en un entorno multidisciplinar. Las competencias elegidas se han trabajado de forma estructurada y coherente, de forma que estas experiencias sirvan posteriormente como ejemplos de buenas prácticas para otros profesores.

\section{Objetivos}

Entendiendo la excelencia desde el punto de vista del desarrollo integral del alumno y de su capacitación para el mundo laboral (GARGALLO,2014), la finalidad del proyecto presentado en esta comunicación es implementar una metodología de enseñanza-aprendizaje, basada en la realización de tareas concretas de aprendizaje y el empleo de determinados instrumentos de evaluación, que permita el desarrollo y la evaluación del grado de adquisición de competencias transversales. Todo ello, con el fin de promover la excelencia en el alumnado.

Para conseguir el objetivo general, se realizará una definición de los niveles de alcance en las competencias transversales de la Universitat Politècnica de València. Esto también supondrá definir los mínimos y los niveles recomendables para cada una de ellas. Hay que tener en cuenta la diversidad de los alumnos, por lo que los niveles de alcance deberán tener una particularización y unos criterios específicos variables según el curso y la titulación, distinguiendo claramente los resultados de aprendizaje esperables para estudios de grado y máster. El objetivo principal del proyecto de innovación que se presenta en esta comunicación es implementar una metodología de enseñanza-aprendizaje que permita el desarrollo y la evaluación del grado de adquisición de competencias transversales en un entorno multidisciplinar. Con el fin de alcanzar este objetivo, se plantean los siguientes objetivos específicos: 
A. Martinez García, M. Cabedo Fabrés, S. Calvet Sanz, A. Jiménez Belenguer, I. Guillén Guillamón, S. Ibánez Asensio, H. Moreno Ramón,C. Cárcel García, M. Ferrando Bataller

- Definir niveles y resultados de aprendizaje esperables en nivel de grado y de master para las competencias transversales estudiadas.

- Establecer tareas específicas para el desarrollo de cada competencia concreta, así como instrumentos para su evaluación.

- Realizar experiencias piloto en las asignaturas participantes, en las que se trate de alcanzar la excelencia en las competencias definidas.

- Demostrar mediante evidencias el nivel de alcance conseguido por los alumnos.

- Proponer un método sistemático que permita trabajar las competencias de forma estructurada y coherente.

- Generar ejemplos de buenas prácticas para otros profesores.

\section{Desarrollo de la innovación}

En esta comunicación se documentan las experiencias piloto llevadas a cabo en diferentes asignaturas en las que se trabajan algunas de las competencias transversales de la UPV, tales como: Compresión e integración, análisis y resolución de problemas, diseño y proyecto, trabajo en equipo y liderazgo, comunicación efectiva, y pensamiento crítico. La metodología de enseñanza aprendizaje propuesta se sustenta en una serie de acciones concretas.

- Determinación de niveles de adquisición de las competencias: para cada una de las competencias se establecerá de forma consensuada unos niveles de alcance tanto para el grado como para el máster, y unos resultados de aprendizaje relativos a cada nivel.

- Realización de un diagnóstico inicial sobre el nivel competencial de los alumnos: al inicio del cuatrimestre los profesores seleccionarán al menos una competencia transversal, de las que se trabajan en la asignatura, utilizando como análisis cuantitativo un estudio de encuesta, en la que se realizará un diagnóstico previo de los alumnos. Esta encuesta inicial completada por los alumnos permitirá conocer el nivel de partida que ellos consideran que poseen en dicha competencia, y al mismo tiempo identificar necesidades concretas y debilidades que presenta ese grupo concreto de alumnos.

- Información a los alumnos del nivel de adquisición de la competencia que deben alcanzar: el nivel de exigencia dependerá del curso/grado/máster que estén cursando. Se les explicará claramente a los alumnos qué nivel se considera deben alcanzar como mínimo, y qué características denotan la excelencia en la adquisición de dicha competencia. 
- Establecimiento de pautas de trabajo para desarrollar la competencia: se darán pautas y directrices claras a los alumnos para la consecución de la competencia mediante documentos, videos, Polimedias, etc. En los casos en los que sea posible se presentarán ejemplos o contraejemplos de buenas prácticas, también se procurará establecer paralelismos con la vida profesional que les permitan valorar la importancia de adquirir la habilidad que se está trabajando.

- Desarrollo de actividades de aprendizaje que permitan adquirir la competencia: cada profesor determinará en base a las peculiaridades de su asignatura y de la competencia a desarrollar una serie de actividades que permitan trabajar la competencia simultáneamente con los contenidos de la asignatura.

- Seguimiento del desarrollo de la competencia: a lo largo del cuatrimestre se irán recogiendo evidencias del grado de consecución de la competencia, mediante sesiones de tutoría, encuestas intermedias, estableciendo puntos de control o realizando pruebas parciales de evaluación.

- Evaluación de la adquisición de la competencia: se emplearán distintas metodologías cuantitativas y cualitativas: rúbricas, pruebas del minuto, evaluación por pares, etc... En aquellas competencias trabajadas en distintas asignaturas se dispondrá de herramientas de evaluación idénticas para poder comparar resultados y la idoneidad de la metodología empleada. También se realizará una encuesta final con respuesta abierta (análisis cualitativo) en la que se investigará la percepción de mejora por parte del alumno, siguiendo con los trabajos desarrollados anteriormente por nuestro grupo y una evaluación de la mejora obtenida al finalizar el mismo.

Como se puede comprobar, la metodología propuesta para desarrollar las competencias transversales está estructurada en acciones claramente pautadas y definidas. Cada profesor cuenta con total libertad a la hora de establecer pautas de trabajo, elegir las actividades de aprendizaje a desarrollar y realizar el seguimiento de la adquisición de la competencia. Esta libertad dota de gran flexibilidad a la metodología propuesta, siendo extrapolable a cualquier disciplina, curso o nivel de estudios.

\section{Resultados}

Para poner en marcha la metodología docente de trabajo de competencias transversales anteriormente descrita, el primer paso consiste en definir niveles de adquisición para cada una de las competencias trabajadas. La Tabla 1 muestra los niveles establecidos por GRIPAU para seis de las competencias transversales definidas por la UPV. Hay que destacar que el nivel 1 se corresponde con el nivel a alcanzar en los primeros dos años de los estudios de grado, el nivel 2 con los cursos tercero y cuarto de estudios de grado, y el nivel 3 con los estudios de Máster. 
A. Martinez García, M. Cabedo Fabrés, S. Calvet Sanz, A. Jiménez Belenguer, I. Guillén Guillamón, S. Ibánez Asensio, H. Moreno Ramón,C. Cárcel García, M. Ferrando Bataller

Tabla 1. Niveles de adquisición establecidos para seis competencias transversales de la UPV

\begin{tabular}{|c|c|}
\hline COMPETENCIA & NIVELES DE ADQUISICIÓN \\
\hline $\begin{array}{l}\text { C1. Comprensión e } \\
\text { integración }\end{array}$ & $\begin{array}{l}\text { Nivel 1. Entender un texto cuando se lee por primera } \\
\text { vez. } \\
\text { Nivel 2. Relacionar conceptos nuevos con otros } \\
\text { aprendidos previamentes y aplicarlos en la resolución } \\
\text { de problemas. } \\
\text { Nivel 3. Aplicación de los conceptos aprendidos a } \\
\text { otros ámbitos. }\end{array}$ \\
\hline $\begin{array}{c}\text { C3. Análisis y resolución de } \\
\text { problemas }\end{array}$ & $\begin{array}{l}\text { Nivel 1. Resolver un ejercicio similar a uno realizado } \\
\text { en clase. } \\
\text { Nivel 2. Resolver un ejercicio abierto aplicando unas } \\
\text { fórmulas proporcionadas. } \\
\text { Nivel 3. Resolver un problema complejo siendo } \\
\text { capaz de deducir la fórmulas a emplear. }\end{array}$ \\
\hline C5. Diseño y proyecto & $\begin{array}{l}\text { Nivel 1. Conocer la estructura de un proyecto y la } \\
\text { metodología para desarrollarlo. } \\
\text { Nivel 2. Desarrollar un proyecto completo partiendo } \\
\text { de cero. } \\
\text { Nivel 3. Ser capaz de replantear el resultado final de } \\
\text { un proyecto frente a otras alternativas consideradas } \\
\text { en proceso de diseño inicial. }\end{array}$ \\
\hline $\begin{array}{c}\text { C6. Trabajo en equipo y } \\
\text { liderazgo. }\end{array}$ & $\begin{array}{l}\text { Nivel 1. Saber valorar las ventajas del trabajo en } \\
\text { equipo, siendo capaz de asumir la responsabilidad } \\
\text { que ello conlleva. } \\
\text { Nivel 2. Conseguir mayor participación e implicación } \\
\text { en la eficacia del grupo } \\
\text { Nivel 3. Potenciar la capacidad de liderazgo, } \\
\text { sabiendo dirigir y marcar objetivos realista al grupo. }\end{array}$ \\
\hline $\begin{array}{l}\text { C8. Comunicación efectiva } \\
\text { (relativo exclusivamente a } \\
\text { comunicación oral) }\end{array}$ & $\begin{array}{l}\text { Nivel 1. Exponer oralmente un problema o trabajo } \\
\text { ante los compañeros de clase, de forma clara y } \\
\text { ordenada. } \\
\text { Nivel 2. Realizar una presentación en público } \\
\text { empleando Power Point o similar. } \\
\text { Nivel 3. Ser capaz de argumentar de forma }\end{array}$ \\
\hline
\end{tabular}




\begin{tabular}{|l|l|}
\hline & organizada las propias ideas en público \\
\hline \multirow{3}{*}{ C9. Pensamiento crítico } & $\begin{array}{l}\text { Nivel 1. Analizar y cuestionar la coherencia de } \\
\text { información, resultados, conclusiones y otros puntos } \\
\text { de vista. } \\
\text { Nivel 2. Debatir con los compañeros o los profesores } \\
\text { otros puntos de vista de forma razonada. } \\
\text { Nivel 3. Llegar a conclusiones y soluciones propias, } \\
\text { y argumentarlas con criterios objetivos ante una } \\
\text { audiencia experta. }\end{array}$ \\
\hline
\end{tabular}

Una vez establecidos de forma consensuada por todos los integrantes del equipo de innovación, los niveles de adquisición a alcanzar por los alumnos, se ha realizado una encuesta inicial que diagnostica el nivel de partida de los alumnos en cada una de las competencias. Hay que destacar que no todas las asignaturas han trabajado todas las competencias, por lo que el número de alumnos implicados en cada competencia es variable. En la Tabla 2 se presentan las competencias analizadas en cada una de las asignaturas involucradas en este proyecto.

Tabla 2. Competencias trabajadas en las asignaturas de la UPV involucradas en este proyecto.

\begin{tabular}{|l|c|c|c|c|c|c|}
\hline Asignatura & C1 & C3 & C5 & C6 & C8 & C9 \\
\hline Teoría de Circuitos & X & X & & X & & \\
\hline $\begin{array}{l}\text { Recuperación y restauración de suelos } \\
\text { degradados }\end{array}$ & $\mathrm{X}$ & $\mathrm{X}$ & & & $\mathrm{X}$ & $\mathrm{X}$ \\
\hline Salud pública & $\mathrm{X}$ & & & & $\mathrm{X}$ & $\mathrm{X}$ \\
\hline Producción de monogástricos & & & & & $\mathrm{X}$ & $\mathrm{X}$ \\
\hline $\begin{array}{l}\text { Sistemas y Servicios de Transmisión por } \\
\text { Radio }\end{array}$ & & & & $\mathrm{X}$ & $\mathrm{X}$ & \\
\hline Eficiencia energética & & & $\mathrm{X}$ & $\mathrm{X}$ & $\mathrm{X}$ & $\mathrm{X}$ \\
\hline Carrera Investigadora & & & & & $\mathrm{X}$ & \\
\hline Comunicaciones Ópticas & & $\mathrm{X}$ & & $\mathrm{X}$ & $\mathrm{X}$ & \\
\hline Sistemas de TV y Video & & $\mathrm{X}$ & & $\mathrm{X}$ & $\mathrm{X}$ & \\
\hline
\end{tabular}


A. Martinez García, M. Cabedo Fabrés, S. Calvet Sanz, A. Jiménez Belenguer, I. Guillén Guillamón, S. Ibánez Asensio, H. Moreno Ramón,C. Cárcel García, M. Ferrando Bataller

Para amenizar la encuesta y facilitar su comprensión por parte de los alumnos, los niveles de adquisición se han traducido en las preguntas relacionadas con situaciones reales en las que los alumnos necesitan hacer uso de las competencias transversales. Además, por simplificar, la respuesta de los alumnos se ha limitado a un simple "Si" o "No". A modo de ejemplo, en la Tabla 3 se muestran las preguntas planteadas al alumno para el diagnóstico inicial de los tres niveles de la competencia C8. Comunicación efectiva.

Tabla 3. Ejemplo de preguntas planteadas al alumno para el diagnóstico inicial de la competencia Comunicación Oral Efectiva.

\begin{tabular}{|l|l|l|l|}
\cline { 3 - 4 } \multicolumn{2}{l|}{ Nivel 1 } & $\begin{array}{l}\text { ¿Has salido alguna vez a la pizarra a } \\
\text { resolver un problema o para explicar } \\
\text { algún concepto? }\end{array}$ & No \\
\hline Nivel 2 & $\begin{array}{l}\text { ¿Has realizado en público una } \\
\text { presentación o trabajo que hayas } \\
\text { preparado previamente? }\end{array}$ & & \\
\hline Nivel 3 & $\begin{array}{l}\text { ¿Has hecho alguna vez una presentación } \\
\text { en público en un ámbito diferente al de } \\
\text { la clase con un debate final sobre los } \\
\text { resultados presentados? }\end{array}$ & & \\
\hline
\end{tabular}

La Fig. 1 resume la valoración inicial de los alumnos respecto a su nivel de partida en las seis competencias transversales objeto de este estudio. Cómo se ha explicado anteriormente, por simplicidad, las respuestas se reducen a SI/NO para cada uno de los niveles en los que se desglosa cada competencia. Es decir, al alumno únicamente responde afirmativa o negativamente a una serie de preguntas relacionadas con sus habilidades. Las respuestas a estas preguntas sirven al profesor para comprobar qué porcentaje de los alumnos tiene dominio de cada competencia antes de cursar la asignatura. La tabla 4 incluye el número de encuestas procesadas en la valoración inicial y final de cada una de las competencia transversales. En total, en el diagnóstico inicial se ha encuestado a 189 alumnos entre las 9 asignaturas implicadas. No obstante, a los alumnos sólo se les ha preguntado por aquellas competencias transversales trabajadas en la asignatura matriculada.

Como se puede observar en la Fig. 1, un elevado porcentaje de alumnos parece tener adquirida la competencia de C1, en los tres niveles establecidos para la misma en la Tabla 1. Igualmente, también se aprecian elevados porcentajes de adquisición en las competencias C5 y C9. La

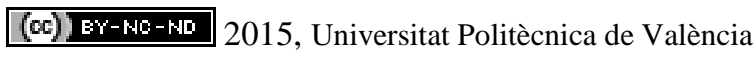

Congreso In-Red (2015) 
competencia en la que los estudiantes parecen tener menos experiencia previa es la C6, ya que un elevado porcentaje de alumnos ha manifestado no saber asumir las responsabilidades que el trabajo en equipo conlleva (Nivel 1), no implicarse en la eficacia del grupo (Nivel 2) y no tener capacidad de liderazgo (Nivel 3). También se observa que un elevado porcentaje de alumnos tiene problemas con el Nivel 3 de la competencia C3 (resolver un problema complejo deduciendo las fórmulas a emplear), y con el Nivel 3 de la competencia C8 (argumentar de forma organizada las propias ideas en público).
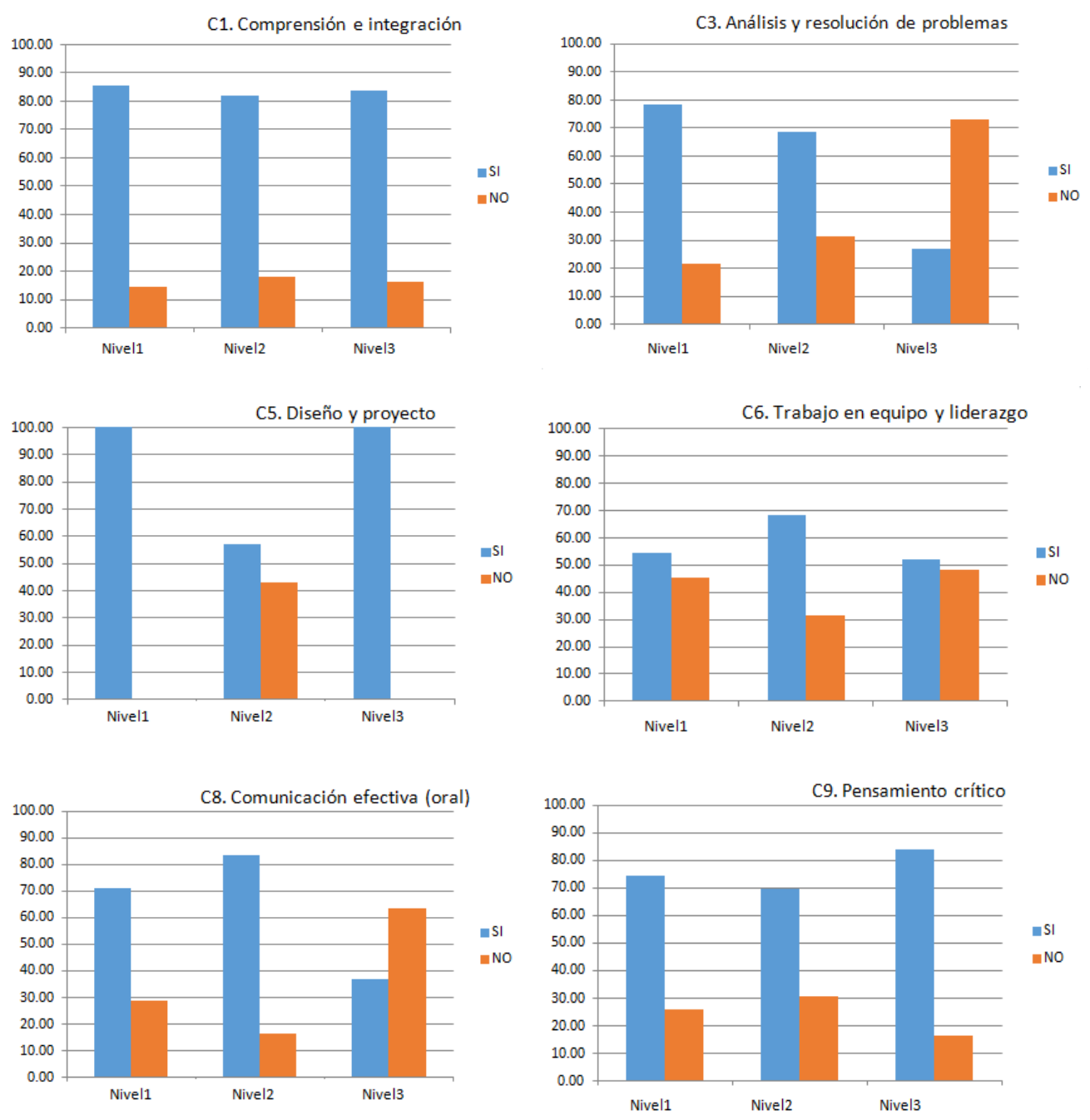

Fig.1. Resultado de la encuesta de valoración inicial de los alumnos respecto a su nivel de partida en las seis competencias transversales objeto de este estudio. 
A. Martinez García, M. Cabedo Fabrés, S. Calvet Sanz, A. Jiménez Belenguer, I. Guillén Guillamón, S. Ibánez Asensio, H. Moreno Ramón,C. Cárcel García, M. Ferrando Bataller

Tabla 4. Número de encuestas iniciales y finales procesadas para cada una de las competencias transversales bajo estudio con un total de 189 (inicial) y 176 alumnos (final) encuestados.

\begin{tabular}{|l|c|c|}
\hline COMPETENCIA & $\begin{array}{c}\mathbf{N}^{\mathbf{0}} \text { de encuestas } \\
\text { procesadas } \\
\text { (inicial) }\end{array}$ & $\begin{array}{c}\text { No de encuestas } \\
\text { procesadas } \\
\text { (final) }\end{array}$ \\
\hline C1. Comprensión e integración & 95 & 111 \\
\hline C3. Análisis y resolución de problemas & 108 & 87 \\
\hline C5. Diseño y proyecto & 7 & 51 \\
\hline C6. Trabajo en equipo y liderazgo. & 77 & 149 \\
\hline $\begin{array}{l}\text { C8. Comunicación efectiva (relativo } \\
\text { exclusivamente a comunicación oral) }\end{array}$ & 164 & 126 \\
\hline C9. Pensamiento crítico & 117 & \\
\hline
\end{tabular}

Los resultados de esta encuesta inicial mostrados en la Fig. 1 se proporcionan a los alumnos para que sean conscientes de su nivel de partida en cada competencia como grupo. A continuación, se les explica qué nivel se considera deben alcanzar como mínimo. El siguiente paso es establecer pautas de trabajo para desarrollar las competencias, para ello se han aprovechado algunos materiales ya existentes (documentos, videos, tutoriales, etc), disponibles a través de Internet o en el repositorio digital institucional de la UPV (Riunet). En algunos casos también se han generado nuevos materiales elaborados por el profesor específicamente para trabajar la competencia al nivel deseado.

Una vez hecho esto, se ha propuesto a los alumnos el desarrollo de una serie de tareas orientadas a mejorar el nivel inicial en la/s competencia/s elegida/s en la asignatura. A modo de ejemplo, se detallan a continuación las propuestas realizadas por las diferentes asignaturas para trabajar la competencia C8. Comunicación Efectiva:

- Asignatura "Recuperación y restauración de suelos degradados", nivel de grado. Los alumnos realizarán un informe técnico que defenderán mediante exposición oral.

- Asignatura "Carrera Investigadora”, de la Escuela de Doctorado, nivel de tercer ciclo. Se realizará un trabajo escrito, siguiendo las pautas indicadas, similares a las que se utilizan en Congresos del ámbito de la Ingeniería. Las evidencias de los trabajos quedarán recogidas en el sitio de PoliformaT, en el apartado de tareas.

(c)) EY-NC-ND 2015, Universitat Politècnica de València

Congreso In-Red (2015) 
- Asignatura "Sistemas y Servicios de Transmisión por Radio", nivel de Máster. Se realizarán cuatro anteproyectos de Sistemas, realizados mediante trabajo en grupo, que serán presentados de forma oral al conjunto de sus compañeros.

- Asignatura "Salud Pública”, nivel de Grado. Mediante la realización de un proyecto: Programa de Educación para la Salud, el cual tienen que presentar en forma de documento escrito y como presentación oral a la clase.

- Asignatura “Eficiencia Energética”, nivel de Máster. A través de la redacción de un proyecto: Intervención de ampliación sobre una vivienda emblemática basada en criterios Medioambientales. La intervención, los criterios en los que se basa y las alternativas planteadas a lo largo del mismo por parte del equipo la deben de presentar en un documento digital de extensión limitada y que conjugue lenguaje gráfico y escrito.

- Asignatura "Producción de Monogástricos”, cuarto curso del Grado en Ingeniería Agronómica y del Medio Rural. Se explica a los alumnos cómo debe realizarse la comunicación escrita y oral en un contexto laboral y se evalúa el grado de adecuación con el cual los alumnos desempeñan estas tareas.

- Asignatura “Comunicaciones Ópticas”, sexto cuatrimestre, nivel de grado. Cada grupo deberá elaborar un documento escrito resolución de los problemas de aplicación real relacionados con los contenidos vistos al finalizar cada uno de los tres bloques temáticos. Posteriormente, cada grupo deberá hacer una exposición oral del problema resuelto con ayuda de los recursos necesarios al profesor en horario de tutorías para poder evaluar la comunicación oral, recursos utilizados, grado adecuación del trabajo realizado.

- Asignatura “Sistemas de TV y Video”, sexto cuatrimestre, nivel de grado. Cada grupo deberá elaborar un documento escrito resolución de los problemas de aplicación real relacionados con los contenidos vistos al finalizar cada uno de los tres bloques temáticos. Posteriormente, cada grupo deberá hacer una exposición oral del problema resuelto con ayuda de los recursos necesarios al profesor en horario de tutorías para poder evaluar la comunicación oral, recursos utilizados, grado adecuación del trabajo realizado.

El seguimiento del desarrollo de la competencia a lo largo del cuatrimestre se ha realizado de forma diferente dependiendo de la asignatura, en unos casos mediante sesiones de tutoría, y en otros estableciendo puntos de control o pruebas parciales de evaluación.

Finalmente, se ha llevado a cabo la evaluación de las competencias empleando elementos diversos como rúbricas, pruebas del minuto o evaluación por pares. También se ha realizado una encuesta final con respuesta abierta (análisis cualitativo) en la que se investiga la percepción de mejora por parte del alumno, siguiendo con los trabajos desarrollados anteriormente por nuestro grupo. La Fig. 2 presenta el resultado de la encuesta de diagnóstico final pasada a los alumnos. Para esta encuesta se ha empleado el mismo conjunto de preguntas utilizadas en la encuesta inicial, que permiten traducir los 
A. Martinez García, M. Cabedo Fabrés, S. Calvet Sanz, A. Jiménez Belenguer, I. Guillén Guillamón, S. Ibánez Asensio, H. Moreno Ramón,C. Cárcel García, M. Ferrando Bataller

niveles de adquisición de cada competencia a situaciones de aplicación con las que se haya enfrentado el alumno. En este caso, las opciones de respuesta están relacionadas con la capacidad del alumno para demostrar la habilidad, dominio o grado de asimilación de la competencia, con una escala: Nunca, a veces, normalmente, o siempre, para cada nivel de la competencia. El número total de encuestas procesadas para cada competencia se puede consultar en la Tabla 4.
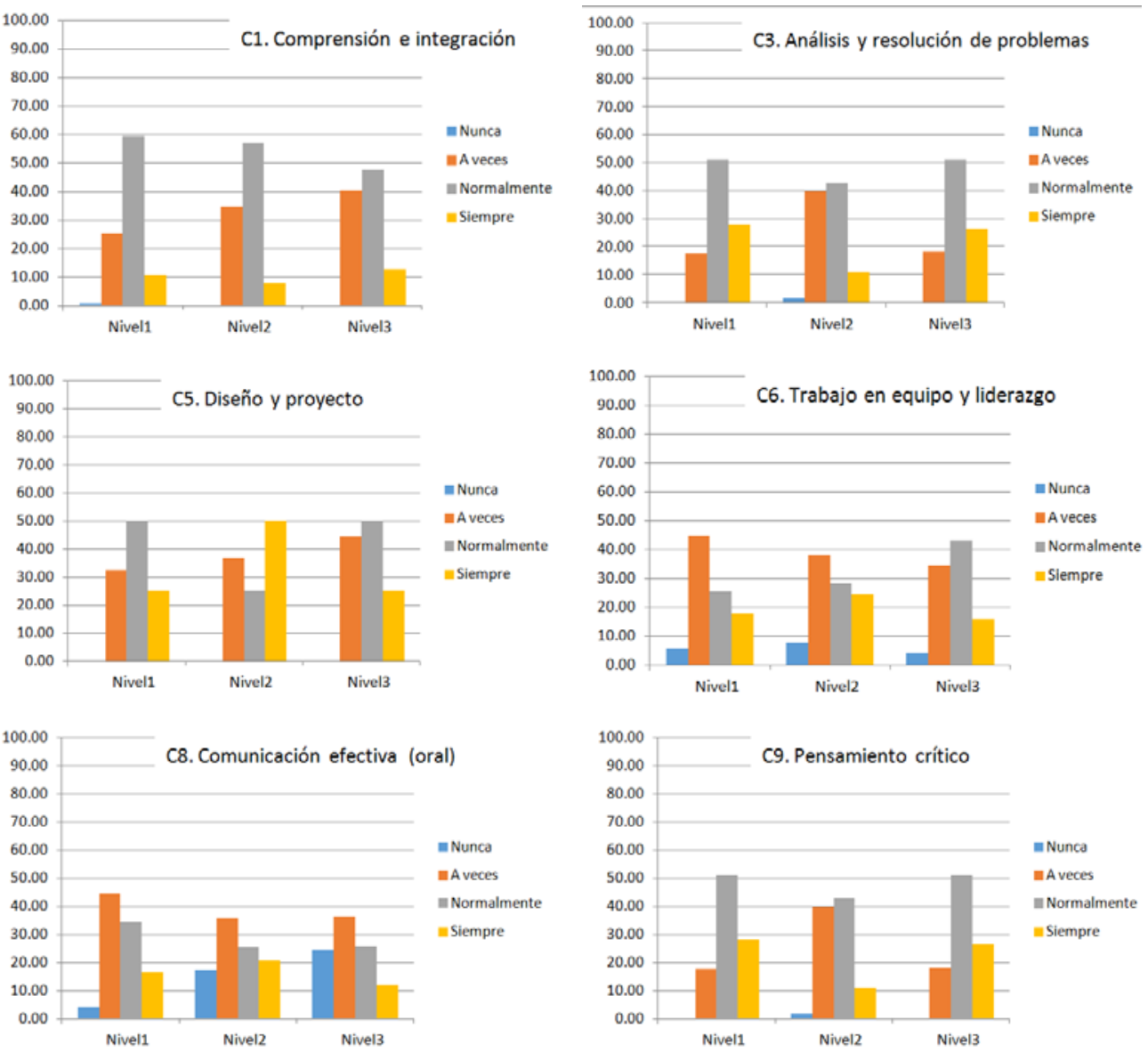

Fig.2. Resultado de la encuesta de diagnóstico final pasada a los alumnos, en la que se les pregunta respecto a su nivel de adquisición en las seis competencias transversales objeto de este estudio.

Observamos que las competencias C1, C5 y C9, en las que se partía de un elevado porcentaje de alumnos con buen nivel de adquisición inicial, presentan excelentes 
resultados en la encuesta final, siendo muy elevado el número de alumnos que es capaz de resolver "normalmente" situaciones relacionadas con la aplicación directa de estas competencias a todos los niveles.

En el caso de la competencia C6 de trabajo en equipo y liderazgo, en la que un elevado porcentaje de alumnos (30\%- 45\%) manifestó no tener ningún nivel en dicha competencia, se observa que la mejora ha sido considerable tras cursar las asignaturas y realizar las tareas establecidas por los profesores. En la encuesta final menos del 7\% ha elegido la opción "nunca" en cualquiera de los niveles, y el porcentaje de alumnos que manifiesta ser capaz de enfrentarse "a veces" y "normalmente" a las situaciones establecidas para los diferentes niveles asciende a aproximadamente el 70\%. Además, entre un 15\% y un 25\% ha elegido la opción "siempre", por lo que puede afirmarse que este grupo ha alcanzado un nivel de excelencia en esta competencia.

En la competencia C3 podemos comprobar que los alumnos poseen buen nivel de adquisición en los niveles 1 y 2, predominando la respuesta "normalmente" (en torno al $50 \%$ ) y destacando el $20 \%$ de respuestas "siempre”. Esto era de esperar, ya que la mayor parte de los alumnos encuestados son de Grado, y son los niveles 1 y 2 los que más se han trabajado en el conjunto de asignaturas seleccionadas. No obstante, inicialmente entre un 20 y un 30\% de los alumnos manifestaba no haber alcanzado estos niveles 1 y 2 , y este porcentaje en la encuesta final pasa a ser prácticamente cero. También hay que destacar que el resultado para el nivel 3 de la competencia C3, que era el más crítico según la encuesta inicial, es ahora francamente positivo. En la encuesta inicial en torno a un $65 \%$ manifestaba no tener adquirido el nivel 3 de la competencia, que como recordaremos estaba relacionado con la resolución de problemas siendo capaz de deducir la fórmulas. Tras ejercitarse realizando problemas de este tipo en las asignaturas cursadas, en la encuesta final se puede comprobar que ahora un $25 \%$ es capaz de enfrentarse a este tipo de problemas "siempre”, un $43 \%$ "normalmente” y un $35 \%$ “ a veces”.

Por último, tenemos la competencia C8 de comunicación efectiva a nivel oral, en la que se observan resultados menos favorables. Llama la atención el elevado porcentaje de respuestas "nunca", $4 \%$ en el nivel 1, y $18 \%$ en el nivel 2. Esto es debido a que en algunas de las asignaturas que cubrían esta competencia, los alumnos han elaborado trabajos de forma grupal, siendo únicamente un representante del grupo el responsable de defender el trabajo oralmente. Esto ha propiciado que algunos alumnos no hayan llegado a trabajar la competencia a lo largo del cuatrimestre, y que hayan marcado la opción "nunca" en la encuesta final. Por otro lado, en el nivel 3 hay un 25\% de respuestas "nunca”, que aunque a primera vista parece un porcentaje elevado, es una gran mejora si lo comparamos con el $63 \%$ de alumnos que inicialmente manifestaba no poseer esta competencia. También es 
A. Martinez García, M. Cabedo Fabrés, S. Calvet Sanz, A. Jiménez Belenguer, I. Guillén Guillamón, S. Ibánez Asensio, H. Moreno Ramón,C. Cárcel García, M. Ferrando Bataller

cierto, que este nivel únicamente se ha trabajado en las asignaturas de nivel de Máster, y que como se ha mencionado anteriormente el porcentaje de alumnos de grado que han completado la encuesta es muy superior al de alumnos de Máster.

\section{Conclusiones}

Los resultados presentados en esta comununicación demuestran que la metodología de enseñanza aprendizaje propuesta permite cuantificar cuantitativa y cualitativamente los distintos niveles y grado de alcance de los mismos por parte de los alumnos en diferentes competencias transversales. La metodología ha sido puesta en práctica por un grupo de profesores multidisciplinar, y en el estudio han participado 189 alumnos de distintas titulaciones. En total se han trabajado y evaluado seis competencias transversales. Se ha verificado que la metodología aplicada permite mejorar el nivel competencial de los alumnos a distintos niveles, siendo extrapolable, dada su gran flexibilidad, a cualquier disciplina, curso o nivel de estudios.

\section{Referencias}

ALSINA, J. (2011). "Evaluación por competencias en la Universidad: las competencias transversales“. Universitat de Barcelona, Institut de Ciències de la Educació. Ediciones Octaedro. 64 p.

AYATS SALT, J.C, JABALOYES VIVAS, J., CAROT SIERRA, J. M., ZAMORA SUÁREZ, J. P., BAENA AROCA, F. M., (2010) "Estudio de empleabilidad de Titulados de la UPV", Editorial Universitat Politècnica de València, Ref. editorial: 2464.

FALLOWS, S., STEVEN, C. (2000). "Building employability skills into the higher education curriculum: a university-wide initiative“. Education + Training 42, 75-82.

FERNÁNDEZ MARCH, A. (2010) "La evaluación orientada al aprendizaje en un modelo de formación por competencias en la educación universitaria”, RED-U, vol 8, nº1, pp. 11-34.

GARGALLO LOPEZ, B., PEREZ PEREZ C. (2014), "Transversal Competences for Employment and Profile of Excellent University Students”, Procedia - Social and Behavioral Sciences, Vol. 139, 22 August 2014, Pages 305-313.

MARTIN DEL PESO, M., RABADAN, A.B., HERNANDEZ-MARCH, J. (2013). “Desajustes entre formación y empleo en el ámbito de las enseñanzas técnicas universitarias: la visión de los empleadores de la Comunidad de Madrid”. Revista de Educación, 360.

TIMES HIGHER EDUCATION WORLD UNIVERSITY RANKINGS (2014), <https://www.timeshighereducation.co.uk/world-university-rankings/2013-14/world-ranking>

UPV (2014). “Plan Estratégico UPV 2015-2020”. Universitat Politècnica de València. p. 43.

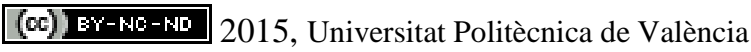

Congreso In-Red (2015) 